\title{
The Psychometric Properties of the Persian Version of the Child and Youth Resilience Measure (CYRM-12)
}

\author{
Susan Ghahremani (iD ${ }^{1}$, Fahimeh Fathali Lavasani (id ${ }^{2,{ }^{*}}$, Mahdieh Moinalghorabaei ${ }^{3}$, Mahmood \\ Dehghani ${ }^{2}$ and Hojjatollah Farahani $\mathbb{( i )}^{4}$ \\ ${ }^{1}$ School of Behavioral Sciences and Mental Health (Tehran Institute of Psychiatry), Iran University of Medical Sciences, Tehran, Iran \\ ${ }^{2}$ Department of Clinial Psychology, School of Behavioral Sciences and Mental Health (Tehran Institute of Psychiatry), Iran University of Medical Sciences, Tehran, Iran \\ ${ }^{3}$ Department of Psychiatry, Faculty of Tehran University of Medical Sciences, Tehran, Iran \\ ${ }^{4}$ Department of Psychology, Faculty of Humanities, Tarbiat Modares University, Tehran, Iran \\ "Corresponding author: Department of Clinial Psychology, School of Behavioral Sciences and Mental Health (Tehran Institute of Psychiatry), Iran University of Medical \\ Sciences, Tehran, Iran. Email: lavasani.f@gmail.com
}

Received 2021 April 21; Revised 2021 June 29; Accepted 2021 September 15.

\begin{abstract}
Background: Resilience is a dynamic system for successful adjustment with various circumstances, particularly adverse living conditions. In this respect, the Child and Youth Resilience Measure (CYRM-12) can simultaneously assess the individual, relational, contextual, and cultural resources of resilience.

Objectives: The present study aimed to investigate the psychometric properties of the Persian version of CYRM-12 in Iranian youth. Methods: In this cross-sectional study, a total number of 440 students aged 14 - 18 years were enrolled. The students were studying in middle and high schools (the academic year of 2019-2020) in the city of Islamshahr, Iran, and were selected using random cluster sampling. Data collection questionnaires included the CYRM-12, CYRM-28, Warwick-Edinburgh Mental Well-Being Scale (WEMWBS), and Depression, Anxiety, and Stress Scale (DASS-21).

Results: Our results supported the one-factor structure and showed that the given measure had a good fit $\left(\chi^{2} / \mathrm{DF}=2.63, \mathrm{RMSEA}=\right.$ $0.06, \mathrm{CFI}=0.95$, and GFI $=0.95$ ). The internal consistency measured by Cronbach's alpha coefficient was also satisfactory (0.79). As well, the test-retest reliability determined by Pearson's correlation coefficient (with a two-week interval) was obtained 0.70. Moreover, this scale had acceptable convergent and divergent validities.
\end{abstract}

Conclusions: The Persian version of the CYRM-12 delivered good reliability and validity to assess resilience in Iranian youth.

Keywords: Children and Youth, CYRM-12, Psychometric, Resilience

\section{Background}

Child and youth development worldwide is threatened by a variety of distresses such as natural disasters, war, poverty, socio-economic problems, family conflicts, and so on (1-3). Although research has so far established that children and youth are at a greater risk of a variety of developmental, emotional, social, behavioral, educational, and psychological problems $(2,4,5)$, many of the individuals facing such distresses (even severe forms for a long time) do not come along with these problems since they are endowed with resilience (6-8).

Resilience is a dynamic system for successful adjustment with different circumstances, particularly adverse living conditions (9). In the past, resilience was merely considered more as a static concept (10), but today, it is regarded as a positive developmental process through which a person facing stressful events (even severe and prolonged distresses) may evade presenting mental illnesses' symptoms $(11,12)$.

Over recent decades, resilience has been among the main topics surrounding the developmental and psychopathological issues of children and the youth. In the first place, the focus has been typically laid on the question of why some children and youths maintain their adjustment despite experiencing adverse conditions such as abuse, neglect, violence, poverty, and deprivation of resources $(9,13,14)$. To address this question, researchers have found that resilience is a multifactorial construct simultaneously built up from individuals' biological, psychological, familial, social, and cultural characteristics, as well as their current and past experiences $(15,16)$. Secondly, researchers have investigated whether resilience can be promoted or taught. In response to this question, there are arguments that the given concept can be learned through- 
out life, or it can even act as a protective factor during child and youth development (17-19).

Youth is a period of life associated with the onset or exacerbation of psychological problems. The consequences of such problems; on the other hand, can have long-term effects on life during adulthood $(20,21)$. Therefore, the youth period can be a good time to learn or strengthen resilience $(22,23)$. In this regard, numerous researchers have investigated the nature of resilience and its determinants and designed programs and interventions to boost resilience in the youth $(24,25)$.

Among the necessities that should be taken into account before developing and implementing resiliencerelated interventions is to design questionnaires that can identify the status of resilience in young people. A review of the previous instruments designed for measuring resilience (like the Connor-Davidson Resilience Scale and Children and Adolescents Resilience Scale [RSCA]) shows that they have focused on the individual characteristics of resilience $(26,27)$, and there is no reliable and validated tool to simultaneously measure individual and socioecological sources of resilience (28). Moreover, a wide range of instruments measure resilience in adulthood while many traumas and adversities occur at younger ages (29).

New definitions of resilience highlight this concept as a construct emphasizing on the interactions between individual and contextual factors in life, bolding the capacity of the youth to exploit health-enhancing resources in culturally meaningful ways (30). Accordingly, Liebenberg, Ungar, and LeBlanc designed a 28-item tool, the Child and Youth Resilience Measure (CYRM-28) (31).

The CYRM-28 has been administered in populations from 11 countries, recommending the questionnaire as an appropriate tool for assessing resilience in different cultures, e.g., Canada, Tanzania, China, Iran, South Africa, etc. (32). Developers have also designed a shorter form of this questionnaire, CYRM-12, to be employed in multifunctional studies and clinical trials. This questionnaire has a one-factor structure and has been validated in two different populations of youths, those affected by adversities and school-aged children. The CYRM-12 can also be used in clinical studies to assess treatment outcome $(28,31)$. In addition, in primary mental health care centers, as a place to screen youths' mental health status and their resilience to stressful conditions, short forms of resilience measures, such as the CYRM-12, can be effective for rapid screening.

In addition to the main study (31), the CYRM-12 has been validated in Arabic (among Lebanese and Syrian refugees) and Chinese cultures. In the Arabic study, it was implemented on 603 refugee and non-refugee youths, delivering good structural and convergent validities (33). The Chinese study was also performed on two samples (i.e., left-behind adolescents and counterparts from different geographical areas of China, with an age range of 9-17), reporting a suitable structural validity (34).

Given the important role of resilience in the promotion of youths' mental health and considering that the CYRM-12 is a questionnaire that can assess resilience from different aspects (viz. individual, relational, communal, and cultural), one question is whether this scale is a good measure for screening of resilience in different cultures or not. Although the structural validity of the CYRM-12 was evaluated by developers in the original study, its other psychometric properties, such as reliability and convergent and divergent validities, need to be further assessed in different populations. Moreover, it can be used in the studies aiming to measure treatment outcomes or those that have multiple goals. Such studies demand for short versions of measures for rapid assessments.

\section{Objectives}

The present study was conducted to investigate the psychometric properties of the Persian version of CYRM-12 in Iranian youths.

\section{Methods}

This study was conducted on a total number of 440 students recruited from middle and high schools (the academic year of 2019 - 2020) in the city of Islamshahr, Iran. The participants' age ranged from 14 to 18 years (mean age $=16.69$, standard deviation $[\mathrm{SD}]=0.94)$, and they included 178 (40.5\%) boys and 262 (59.5\%) girls. To collect the data, a list of young students studying at the schools of Islamshahr was initially prepared. Then eight schools from different regions with various cultural and socioeconomic backgrounds were selected using random cluster sampling. After that, one classroom was randomly selected from each school, and the research questionnaires were administered to the students. In addition, 181 youths completed the research questionnaires twice (with a two-week interval) to measure its reliability using the internal consistency and test-retest methods.

Inclusion criteria were an age range between 11 and 18 years, fluency in the Persian language, as well as having motivation and willingness to participate in the study. The number of the participants was selected based on a survey by Comrey and Lee (35), who reported that 200500 individuals were adequate for factor analysis. In the present study, 440 participants were selected to increase the study's validity. Exclusion criteria included incompletely filling the questionnaire or failure to answer more than $10 \%$ of the questions. 


\subsection{Measures}

\subsubsection{The child and Youth Resilience Measure (28-item)}

The CYRM-28 $(28,36)$ has been designed for the ecological measurement of resilience in the youth aged 12-23 years. This measure contains 28 items and examines individual, relational, communal, and cultural resources of resilience. Participants can answer each question on a five-point Likert scale ranging from one to five. The minimum and maximum scores in this questionnaire are 28 and 140 , respectively. A higher score indicates a higher resilience. The reliability coefficients of the Persian version of this questionnaire in various dimensions have been reported from 0.71 to 0.85 (37).

\subsubsection{The child and Youth Resilience Measure (12-item)}

The CYRM-12 is a self-report scale derived from the CYRM-28 and is often used for the ages of $10-23$ years. Respondents mark their agreement with each item on a Likert-type scale from not at all (1) to a lot (5). The minimum and maximum scores in this questionnaire are 12 and 60 , respectively, and a higher score represents more resilience. In the original study by the developers, the reliability coefficient of this questionnaire via the internal consistency method was reported 0.84 (31).

\subsubsection{Depression, Anxiety and Stress Scale}

The Depression, Anxiety and Stress Scale (DASS-21) is a 21-item self-report questionnaire that measures depression, stress, and anxiety in individuals. Each scale also contains seven items with four options graded on a scale from zero (did not apply to me at all) to three (applied to me much or most of the time). The internal consistency of the total score of the scale was 0.93 , and the respective values were $0.82,0.90$, and 0.93 for the scales of depression, anxiety, and stress, respectively (38). Also, the internal consistency coefficients of the Persian version of DASS-21 were $0.77,0.79$, and 0.78 for depression, anxiety, and stress, respectively (39).

\subsubsection{Warwick-Edinburgh Mental Well-being Scale}

The Warwick-Edinburgh Mental Well-Being Scale (WEMWBS) examines the mental well-being of individuals over the past two weeks and contains 14 items and three dimensions (viz. positive effects, satisfying interpersonal relationships, and positive functioning). Each item is also scored using a Likert-type scale from one to five. The total score of the questionnaire is obtained by summing up the scores of the dimensions and ranges from 14 to 70 . Cronbach's alpha coefficients of the student and general population versions of the scale have been reported 0.89 and 0.91 , respectively. As well, test-retest reliability within a one-week period has been equal to 0.83 (40). Cronbach's coefficient of the Persian version of the scale was reported $0.78(41)$.

\subsection{Procedure}

The following steps were taken to translate the CYRM-12 according to the guidelines for translating questionnaires in intercultural studies (42). At the first stage, after sending an email to its developers and obtaining their permission, its English version was translated to Persian by two Ph.D. students in clinical psychology who were fluent in English and Persian. Then backward translation was done by an English language expert familiar with Persian. Finally, the original and the backward English translations were sent to the developers, and after their approval, the CYRM-12, CYRM-28, WEMWBS, and DASS-21 were provided to the participants.

\subsection{Ethical Considerations}

Ethical approval was obtained from the university's research committee (IR.IUMS.REC.1398.695). The students were invited to participate in the study, and they were informed that their participation was voluntary and knew that they could discontinue at any time. The participants were also ensured about confidentiality.

\section{Results}

Confirmatory factor analysis (CFA) was used to investigate the structural validity of the CYRM-12 questionnaire. Moreover, its convergent and divergent validities were checked with regard to the CYRM-28, DASS-21, and WEMWBS. Finally, the data were analyzed using descriptive statistics, correlation coefficients, and CFA in SPSS (ver. 26) and LISERL 8.8 software.

\subsection{Confirmatory Factor Analysis}

To perform CFA, a one-factor structural model was hypothesized for the questionnaire (Figure 1). The model's evaluation was based on considering a variety of fit indices such as chi-square $(<3)$, standardized root mean square residual(SRMR), root mean square error of approximation (RMSEA) (< 0.08), comparative fit index (CFI), normed fit index (NFI), relative fit index (RFI), and goodness of fit index (GFI) (a range from 0 to 1 and a value of 0.90 or greater indicating a good fitting model) (43-45). The results of the model fit indices have been presented in Table 1, confirming the suitability of the model. 


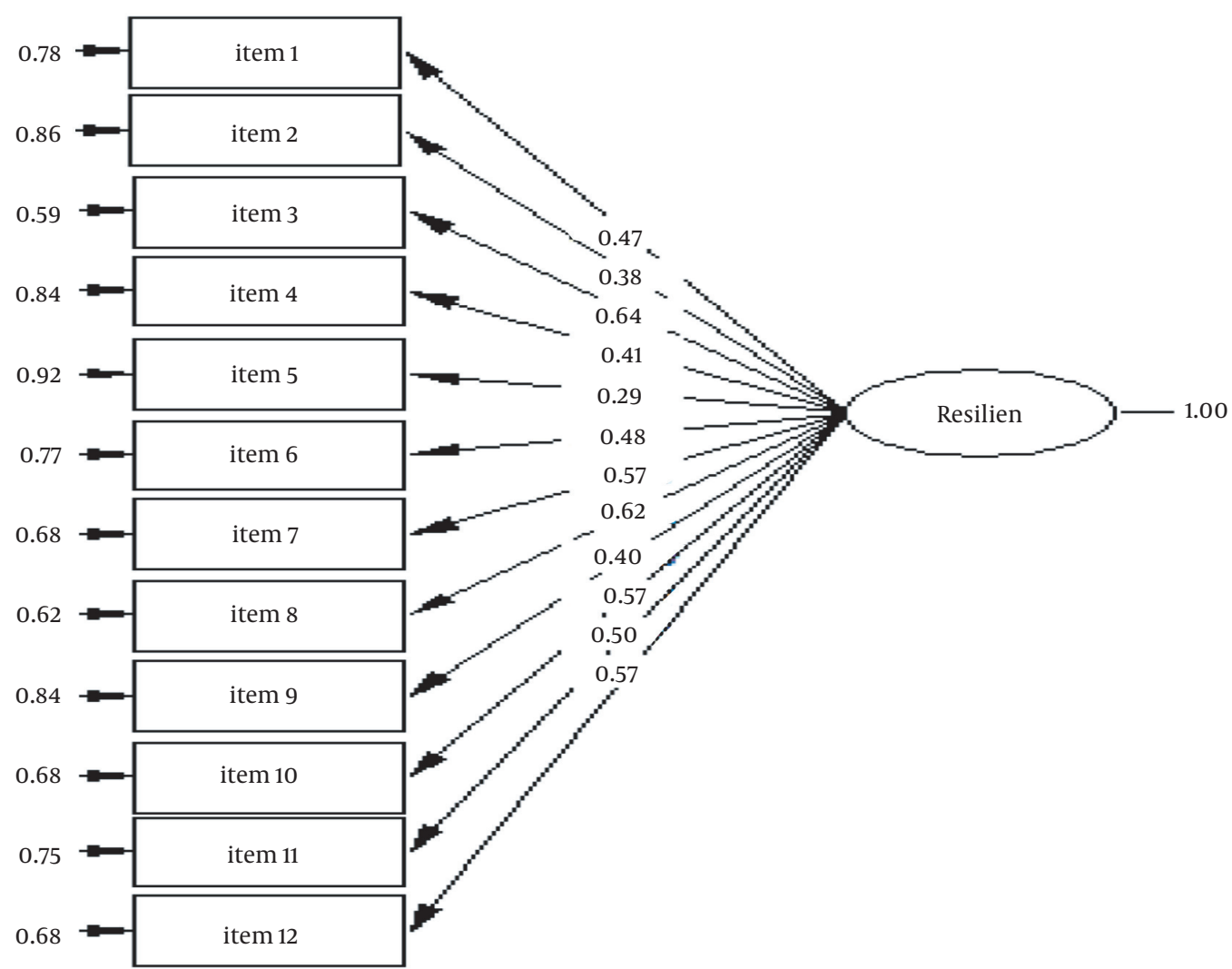

Figure 1. Confirmatory Factor Analysis for CYRM-12

Tabel 1. Fit Indices for the One-factor Model

\begin{tabular}{ccccccccccc}
\hline \multicolumn{10}{c}{} & \multicolumn{10}{c}{ Fit Indexes } \\
\cline { 2 - 10 } & $\mathbf{P}$ & $\chi^{\mathbf{2}} / \mathbf{d f}$ & SRMR & GFI & IFI & RFI & AGFI & NNFI & NFI & RMSEA \\
\hline Resiliency 2.29 & 0.001 & 2.63 & 0.05 & 0.95 & 0.95 & 0.91 & 0.93 & 0.93 & 0.94 & 0.06 \\
\hline
\end{tabular}

\subsection{Convergent and Divergent Validities}

To measure the divergent and convergent validities of the CYRM-12, all three CYRM-28, WEMWBS, and DASS-21 questionnaires were employed. Pearson's correlation coefficient was also calculated between the scores of these questionnaires and that of CYRM-12 (Table 2). In this sense, correlations between the scores of CYRM-12, CYRM-28, and WEMWBS were positive and significant, suggesting a good convergent validity. The correlation with DASS-21 was also negative and significant, implying a good divergent validity.

\subsection{Reliability}

The reliability of CYRM-12 was established in two ways. First, the internal consistency of the questionnaire was calculated using Cronbach's alpha coefficient, which was ob- tained 0.79. Secondly, the test-retest reliability was measured according to intraclass correlation coefficient (ICC), which was 0.70 . These values indicated that the reliability of the questionnaire was good (46).

\subsection{Gender Comparisons}

The results of the independent samples student $t$-test showed no significant difference between boys and girls in terms of the mean scores of CYRM-12 (t-statistic (438) = -0.04 , Sig. $=0.966)$.

\section{Discussion}

New definitions of resilience regard this entity as a multifactorial construct affected by individual, relational, contextual, and cultural resources. Considering the role of resilience as a protective factor during the development of children and youths in reducing vulnerability to physical and psychological problems, Leibenberg, Ungar, and Lee Blanche developed the CYRM-12 to assess resilience in these populations (31). The aim of the present study was to investigate some of CYRM-12 psychometric properties (such 


\begin{tabular}{lcccc}
\hline Table 2. The Convergent and Divergent Validities of CYRM-12 ${ }^{\text {a }}$ & & & \\
\hline Variables & 1 & & & \\
\hline CYRM-12 & $0.64^{* *}$ & & & \\
Mental well-being & $-0.42^{* *}$ & $-0.45^{* *}$ & 1 & 1 \\
Depression & $-0.29^{* *}$ & $-0.32^{* *}$ & $0.64^{* *}$ & $0.72^{* *}$ \\
\hline Stress & $-0.34^{* *}$ & $-0.34^{* *}$ & $0.66^{* *}$ & 1 \\
\hline Anxiety & & & \\
\hline
\end{tabular}

$\mathrm{a} * \mathrm{P}<0.05,{ }^{* *} \mathrm{P}<0.01$

as convergent and divergent validities, concurrent validity, and test-retest reliability) that were not examined in the original study by the developers. In addition, some other psychometric properties, such as construct validity, reliability (measured by the internal consistency method), and gender differences in resilience, were assessed in Iranian youths.

In this regard, CFA results supported the one-factor structure of the tool and showed that the given model had a good fit. In addition, the GFI values obtained in this study were similar to those obtained in the original study (31) and were consistent with the outcomes of a Chinese study on school-age youths (34). However, contrary to the results of the three above-mentioned studies, including ours, in a Lebanese study on Syrian refugees and Lebanese youths, CFA results based on CYRM-12 items were poor, and the researchers in this study simply validated another version of CYRM-12 that had a good fit (33). The difference in the GFI value of the one-factor model in the studies conducted in Canada, China, and Iran compared with that of the Lebanese study can be related to variations in the type of samples. Unlike the first three studies who enrolled the youths experiencing no major crisis in lifetime, the Lebanese study was performed on the Syrian youths who had experienced war traumas and had taken refuge in Lebanon.

The reliability of CYRM-12 in this study was further determined using Cronbach's alpha coefficient, which was 0.79 and lower than that reported in the original study by the developers $(\alpha=0.84)$ (31) but still showed a good reliability. In the Chinese study, the researchers also reported a higher Cronbach's alpha coefficient (0.92) compared to ours (34). Compared with the reliability obtained in the Syrian refugees (0.75) and Lebanese youths (0.75), the internal consistency of the Persian version was slightly higher (33). The examination of CYRM-12 stability within a twoweek interval showed a correlation coefficient of 0.70 , indicating a good value. In comparison with the present study, the study on Syrian and Lebanese refugee youths reported a one-week stability of 0.93 for CYRM-12 (33). The lower value in our study was justifiable in respect of our longer time interval.

The strong correlation $(\mathrm{r}=0.86, \mathrm{P}<0.05)$ between CYRM-12 and CYRM-28 indicated the high convergent validity of these two questionnaires. As expected, CYRM-12 showed a significantly negative correlation with the symptoms of depression, anxiety, and stress and a significant but positive correlation with mental well-being. These results were consistent with the findings of a study by Kelly et al. (47), as well as another study by Hjemdal et al. (48), who found that boosting resilience was associated with reduced symptoms of depression, anxiety, and stress, which was in line with the findings of the present study. In another survey, researchers (49) showed that individuals with high degrees of resilience had higher levels of mental well-being components, such as positive cognition and life satisfaction, and lower levels of depression. In fact, resilient people could show positive emotions in the face of adversities, and resilience could be a predictor of youth well-being (50). In support of these findings, Davydov et al. (17) reported that resilience was an important defense mechanism to mitigate vulnerability to mental health problems and to maintain or enhance mental well-being.

The comparison of resilience between the genders indicated no significant difference between boys and girls. These findings were consistent with the results reported by Panter-Brick et al. (33), Zand et al. (37), and Ghannam and Thabet (51); all of which had been conducted in Asian countries. In contrast with this study, some studies reported that resilience was different between boys and girls (52), indicating the importance of gender in determining resilience in the youth, depending on whether the group has experienced traumas or not or what resilience sources (namely, individual, relational, communal, or cultural) have been examined in each study (or questionnaire). In boys and girls, different individual and communal factors seem to affect resilience, explaining the gender differences observed in resilience $(37,53-56)$.

The present study had some limitations. Even though 
the psychometric properties of the CYRM-12 questionnaire were evaluated in the youth, the study sample merely included normal youths, and those with clinical conditions were not examined. Therefore, it is suggested to recruit clinical groups in future surveys. In addition, the demographic characteristics of the population should be considered when generalizing the findings, so another limitation was that the data of the present study were collected from only one city in Iran. In this study, the age of the participants ranged from 14 to 18 years, but future research should reflect on other age groups, for example, 11-23-yearold.

\subsection{Conclusions}

The results of this study revealed that the CYRM-12 questionnaire had good construct, convergent, and divergent validities, as well as a good reliability. Therefore, the Persian version of CYRM-12 can be used to determine the degree of resilience in Iranian youths. Further research is needed to investigate the reliability and validity of CYRM12 , as a screening tool, in other Iranian populations, as well as in clinical populations, to assess treatment outcomes.

\section{Footnotes}

Authors' Contribution: Fahimeh Fathali Lavasani, Susan Ghahremani, and Mahdiyeh Moinalghobrabaie designed the study; Hojatollah Farahaneie conducted statistical analysis; Fahimeh Fathali Lavasani and Susan Ghahremani contributed to data collection; Fahimeh Fathali Lavasani, Susan Ghahremani, and Mahmood Dehghani wrote the first draft of the manuscript. All authors read and approved the content of the final manuscript.

Conflict of Interests: The authors declare that they have no conflict of interest.

\section{Ethical Approval: IR.IUMS.REC.1398.695.}

Funding/Support: Iran University of Medical Sciences supported the study.

Informed Consent: All individual participants were informed about the study aims and were asked to complete the questionnaires only if they were willing to participate in the study.

\section{References}

1. Kuhlman KR, Robles TF, Bower JE, Carroll JE. Screening for childhood adversity: the what and when of identifying individuals at risk for lifespan health disparities.J Behav Med. 2018;41(4):516-27. eng. doi: 10.1007/s10865-018-9921-z. [PubMed: 29603040]. [PubMed Central: PMC6031456].

2. Wickrama KA, Lee TK, O'Neal CW. Stressful life experiences in adolescence and cardiometabolic risk factors in young adulthood.J Adolesc Health. 2015;56(4):456-63. eng. doi: 10.1016/j.jadohealth.2014.12.009. [PubMed: 25659202].
3. Kuhlman KR, Horn SR, Chiang JJ, Bower JE. Early life adversity exposure and circulating markers of inflammation in children and adolescents: A systematic review and meta-analysis. Brain Behav Immun. 2020;86:30-42. eng. doi: 10.1016/j.bbi.2019.04.028. [PubMed: 30999015]. [PubMed Central: PMC8211400].

4. Kysar-Moon A. Childhood adversity and internalizing problems: Evidence of a race mental health paradox. Soc Ment Health. 2020;10(2):136-62.

5. Nelson CA, Scott RD, Bhutta ZA, Harris NB, Danese A, Samara M. Adversity in childhood is linked to mental and physical health throughout life. Bmj. 2020;371:m3048. eng. doi: 10.1136/bmj.m3048. [PubMed: 33115717]

6. Malhi GS, Das P, Bell E, Mattingly G, Mannie Z. Modelling resilience in adolescence and adversity: a novel framework to inform research and practice. Transl Psychiatry. 2019;9(1):316. eng. doi: 10.1038/s41398019-0651-y. [PubMed: 31772187].

7. Galatzer-Levy IR, Huang SH, Bonanno GA. Trajectories of resilience and dysfunction following potential trauma: A review and statistical evaluation. Clin Psychol Rev. 2018;63:41-55. eng. doi: 10.1016/j.cpr.2018.05.008. [PubMed: 29902711].

8. Jin J, Tang YY, Ma Y, Lv S, Bai Y, Zhang H. A structural equation model of depression and the defense system factors: a survey among Chinese college students. Psychiatry Res. 2009;165(3):288-96. eng. doi: 10.1016/j.psychres.2008.03.012. [PubMed: 19168228].

9. Masten AS. Global perspectives on resilience in children and youth. Child Dev. 2014;85(1):6-20. eng. doi: 10.111//cdev.12205. [PubMed: 24341286].

10. Pooley JA, Cohen L. Resilience: A definition in context.Aust Community Psychol.2010;22(1):30-7.

11. Kalisch R, Müller MB, Tüscher O. A conceptual framework for the neurobiological study of resilience. Behav Brain Sci. 2015;38. eng. e92. doi: 10.1017/s0140525x1400082x. [PubMed: 25158686].

12. Masten AS, Best K, Garmezy N. Resilience and development: Contributions from the study of children who overcome adversity. Dev Psychopathol.1990;2(4):425-44. doi: 10.1017/S0954579400005812.

13. Cicchetti D. Annual Research Review: Resilient functioning in maltreated children-past, present, and future perspectives. $J$ Child Psychol Psychiatry. 2013;54(4):402-22. eng. doi: 10.1111/j.14697610.2012.02608.x. [PubMed: 22928717]. [PubMed Central: PMC3514621].

14. Masten AS. Resilience in children threatened by extreme adversity: frameworks for research, practice, and translational synergy. Dev Psychopathol. 2011;23(2):493-506. eng. doi: 10.1017/s0954579411000198. [PubMed: 23786691].

15. Wu G, Feder A, Cohen H, Kim JJ, Calderon S, Charney DS, et al. Understanding resilience. Front Behav Neurosci. 2013;7:10. eng. doi: 10.3389/fnbeh.2013.00010. [PubMed: 23422934]. [PubMed Central: PMC3573269].

16. Cicchetti D. Resilience under conditions of extreme stress: a multilevel perspective. World Psychiatry. 2010;9(3):145-54. eng. doi: 10.1002/j.2051-5545.2010.tb00297.x. [PubMed: 20975856]. [PubMed Central: PMC2948722].

17. Davydov DM, Stewart R, Ritchie K, Chaudieu I. Resilience and mental health. Clin Psychol Rev. 2010;30(5):479-95. eng. doi: 10.1016/j.cpr.2010.03.003. [PubMed: 20395025].

18. Zhao X, Fu F, Zhou L. The mediating mechanism between psychological resilience and mental health among left-behind children in China. Child Youth Serv Rev. 2020;110:104686. doi: 10.1016/j.childyouth.2019.104686.

19. Hughes K, Bellis MA, Hardcastle KA, Sethi D, Butchart A, Mikton C, et al. The effect of multiple adverse childhood experiences on health: a systematic review and meta-analysis. Lancet Public Health. 2017;2(8):e35666. eng. doi: 10.1016/s2468-2667(17)30118-4. [PubMed: 29253477]. 
20. Ogden T, Hagen KA. Adolescent mental health: Prevention and intervention. Routledge; 2018.

21. Das JK, Salam RA, Lassi ZS, Khan MN, Mahmood W, Patel V, et al. Interventions for Adolescent Mental Health: An Overview of Systematic Reviews. J Adolesc Health. 2016;59(4s):S49-s60. eng. doi: 10.1016/j.jadohealth.2016.06.020. [PubMed: 27664596]. [PubMed Central: PMC5026677].

22. Hildebrand NA, Celeri E, Morcillo AM, Zanolli ML. Resilience and mental health problems in children and adolescents who have been victims of violence. Rev Saude Publica. 2019;53:17. doi: 10.11606/s1518-8787.2019053000391. [PubMed: 30726498]. [PubMed Central: PMC6390680].

23. Soleimanpour S, Geierstanger S, Brindis CD. Adverse Childhood Experiences and Resilience: Addressing the Unique Needs of Adolescents. Acad Pediatr. 2017;17(7s):S108-s114. eng. doi:10.1016/j.acap.2017.01.008. [PubMed: 28865641].

24. Dray J, Bowman J, Campbell E, Freund M, Wolfenden L, Hodder RK, et al. Systematic Review of Universal Resilience-Focused Interventions Targeting Child and Adolescent Mental Health in the School Setting. J Am Acad Child Adolesc Psychiatry. 2017;56(10):813-24. eng. doi: 10.1016/j.jaac.2017.07.780. [PubMed: 28942803].

25. Scarf D, Hayhurst JG, Riordan BC, Boyes M, Ruffman T, Hunter JA. Increasing resilience in adolescents: the importance of social connectedness in adventure education programmes. Australas Psychiatry. 2017;25(2):154-6. eng. doi: 10.1177/1039856216671668. [PubMed: 27679628].

26. Connor KM, Davidson JR. Development of a new resilience scale: the Connor-Davidson Resilience Scale (CD-RISC). Depress Anxiety. 2003;18(2):76-82. eng. doi: 10.1002/da.10113. [PubMed: 12964174].

27. Prince-Embury S, Courville T. Comparison of one-, two-, and threefactor models of personal resiliency using the resiliency scales for children and adolescents. Can J Sch Psychol. 2008;23(1):11-25. doi: $10.1177 / 0829573508316589$.

28. Liebenberg L, Ungar M, Vijver FVD. Validation of the child and youth resilience measure-28(CYRM-28) among Canadian youth. Res Soc Work Pract. 2012;22(2):219-26. doi: 10.1177/1049731511428619.

29. Miller-Graff LE, Cummings EM. The Israeli-Palestinian conflict: Effects on youth adjustment, available interventions, and future research directions. Dev Rev. 2017;43:1-47. doi: 10.1016/j.dr.2016.10.001.

30. Ungar M. Resilience across Cultures. BrJ Soc Work. 2006;38(2):218-35. doi: 10.1093/bjsw/bcl343.

31. Liebenberg L, Ungar M, LeBlanc JC. The CYRM-12: a brief measure of resilience. Can J Public Health. 2013;104(2):e131-5. eng. doi: 10.1007/bf03405676. [PubMed: 23618205]. [PubMed Central: PMC6974279].

32. Ungar M, Liebenberg L. The Child and Youth Resilience Measure (CYRM) Child Version. Users Manual. Child Outcomes Research Consortium; [updated 2016]. Available from: www.corc.uk.net/outcomeexperience-measures/child-and-youth-resilience-measurechildversion.

33. Panter-Brick C, Hadfield K, Dajani R, Eggerman M, Ager A, Ungar M. Resilience in Context: A Brief and Culturally Grounded Measure for Syrian Refugee and Jordanian Host-Community Adolescents. Child Dev. 2018;89(5):1803-20. eng. doi: 10.1111/cdev.12868. [PubMed: 28617937]. [PubMed Central: PMC6208286].

34. Mu GM, Hu Y. Validation of the Chinese version of the 12-item child and youth resilience measure. Child Youth Serv Rev. 2016;70:332-9. doi: 10.1016/j.childyouth.2016.09.037.

35. Comrey A, Lee H, Comrey AL, Lee HB. Interpretation and application of factor analytic results. A first course in factor analysis. 2. Psychology Press; 1992.

36. Ungar $M$, Liebenberg L. Assessing resilience across cultures using mixed methods: Construction of the child and youth resilience measure. J Mix Methods Res. 2011;5(2):126-49. doi: $10.1177 / 1558689811400607$.

37. Zand BK, Liebenberg L, Shamloo ZS. Validation of the factorial struc- ture of the Child and Youth Resilience Measure for use with Iranian youth. Child Indic Res. 2017;10(3):797-809. doi: 10.1007/s12187-016-94120 .

38. Henry JD, Crawford JR. The short-form version of the Depression Anxiety Stress Scales (DASS-21): construct validity and normative data in a large non-clinical sample. Br J Clin Psychol. 2005;44(Pt 2):227-39. eng. doi: 10.1348/014466505x29657. [PubMed: 16004657].

39. Sahebi A, Asghari MJ, Salari RS. Validation of depression anxiety and stress scale (DASS-21) for an Iranian population. Iran J Psychol. 2005.

40. Tennant R, Hiller L, Fishwick R, Platt S, Joseph S, Weich S, et al. The Warwick-Edinburgh Mental Well-being Scale (WEMWBS): development and UK validation. Health Qual Life Outcomes. 2007;5:63. eng. doi: 10.1186/1477-7525-5-63. [PubMed: 18042300]. [PubMed Central: PMC2222612].

41. Rajabi G. [Validity and Reliability of the Persian Version of the Mental Well-Being Scale in Cancer Patients. Quart J Health Psychol]. 2013;1(4):30-41. Persian.

42. Guillemin F, Bombardier C, Beaton D. Cross-cultural adaptation of health-related quality of life measures: literature review and proposed guidelines. J Clin Epidemiol. 1993;46(12):1417-32. eng. doi: 10.1016/0895-4356(93)90142-n. [PubMed: 8263569].

43. Brown TA. Confirmatory factor analysis for applied research. Guilford publications; 2015.

44. Tabachnick BG. BG Tabachnick, LS Fidell using multivariate statistics. Boston: Pearson; 2013.

45. Hu L, Bentler PM. Cutoff criteria for fit indexes in covariance structure analysis: Conventional criteria versus new alternatives. Struct Equ Modeling. 1999;6(1):1-55. doi: 10.1080/10705519909540118.

46. Hair J, Black W, Babin B, Anderson R. Multivariate data analysis: Pearson new. UK: International edition Harlow; 2014.

47. Kelly Y, Fitzgerald A, Dooley B. Validation of the Resilience Scale for Adolescents (READ) in Ireland: a multi-group analysis. Int J Methods Psychiatr Res. 2017;26(2). eng. doi: 10.1002/mpr.1506. [PubMed: 27126561]. [PubMed Central: PMC6877176].

48. Hjemdal O, Vogel PA, Solem S, Hagen K, Stiles TC. The relationship between resilience and levels of anxiety, depression, and obsessivecompulsive symptoms in adolescents. Clin Psychol Psychother. 2011;18(4):314-21. eng. doi:10.1002/cpp.719. [PubMed: 20806419].

49. Mak WW, Ng IS, Wong CC. Resilience: enhancing well-being through the positive cognitive triad.J Couns Psychol. 2011;58(4):610-7. eng. doi: 10.1037/a0025195. [PubMed: 21895357].

50. Vinayak S, Judge J. Resilience and empathy as predictors of psychological wellbeing among adolescents. Int JHealth Sci Res. 2018;8(4):192200.

51. Ghannam RT, Thabet A. Effect of trauma due to war on dissociative symptoms and resilience among Palestinian adolescents in the Gaza Strip. Arab J Psychiatry. 2014;25(2):107-18. doi:10.12816/0006760.

52. Stratta P, Capanna C, Patriarca S, de Cataldo S, Bonanni RL, Riccardi I, et al. Resilience in adolescence: Gender differences two years after the earthquake of L'Aquila. Pers Individ Dif. 2013;54(3):327-31. doi: 10.1016/j.paid.2012.09.016.

53. Mistry R, McCarthy WJ, Yancey AK, Lu Y, Patel M. Resilience and patterns of health risk behaviors in California adolescents. Prev Med. 2009;48(3):291-7. eng. doi: 10.1016/j.ypmed.2008.12.013. [PubMed: 19159644]. [PubMed Central: PMC2692484].

54. Amstadter AB, Myers JM, Kendler KS. Psychiatric resilience: longitudinal twin study. Br J Psychiatry. 2014;205(4):275-80. eng. doi: 10.1192/bjp.bp.113.130906. [PubMed: 24723629]. [PubMed Central: PMC4180845]

55. Newsome J, Vaske JC, Gehring KS, Boisvert DL. Sex Differences in Sources of Resilience and Vulnerability to Risk for Delinquency. $J$ Youth Adolesc. 2016;45(4):730-45. eng. doi: 10.1007/s10964-015-0381-2. [PubMed: 26525388].

56. Namy S, Carlson C, Norcini Pala A, Faris D, Knight L, Allen E, et al. Gender, violence and resilience among Ugandan adolescents. Child Abuse 
